\title{
SOLVABILITY OF DISCONTINUOUS FUNCTIONAL DIFFERENTIAL SYSTEMS IN $l_{\infty}(M)$
}

\author{
A. CABADA $\llbracket 1$, J. ÁNGEL CID $^{2}$ and S. HEIKKIL $\ddot{A}^{3}$
}

(Received 23 February, 2005; revised 21 August, 2006)

\begin{abstract}
We study the existence of extremal solutions for an infinite system of first-order discontinuous functional differential equations in the Banach space of the bounded functions $l_{\infty}(M)$.
\end{abstract}

2000 Mathematics subject classification: primary 34A36, 34K07, 47H07.

Keywords and phrases: extremal solutions, functional differential system, discontinuous nonlinearities.

\section{Introduction}

The study of functional differential equations covers, among others, ordinary differential equations, integro-differential equations and equations with maxima. Moreover these types of equations appear when we use the reduction of order method for a suitable scalar $n$ th-order ordinary differential equation, [2,4], which can obviously be treated as a first-order system of finite equations. Following this idea, one can consider differential functional systems with infinitely many equations, the number of which is not necessarily countable. Such systems have been studied by several different authors, see [7] and the references therein.

In this paper we study the solvability of an infinite system of functional differential equations, with nonlinear functional boundary value conditions, in the Banach space

\footnotetext{
${ }^{1}$ Departamento de Análise Matemática, Facultade de Matemáticas, Campus Sur, Universidade de Santiago de Compostela, 15782 Santiago de Compostela, Spain; e-mail: cabada@usc.es.

${ }^{2}$ Departamento de Matemáticas, E.U.P. de Linares, Universidad de Jaén C / Alfonso X el Sabio, no. 28, 23700, Linares, Jaén, Spain; e-mail: angelcid@ujaen.es.

${ }^{3}$ Department of Mathematical Sciences, University of Oulu, Box 3000, FIN-90014, University of Oulu, Finland; e-mail: sheikki@cc.oulu.fi.

(C) Australian Mathematical Society 2006, Serial-fee code 1446-1811/06
} 
of the bounded functions $l_{\infty}(M)$, where $M$ is an arbitrary index set

$$
\begin{cases}u_{v}^{\prime}(t)=g_{\nu}(t, u(t), u) & \text { for a.a. } t \in I:=\left[t_{0}, t_{1}\right], v \in M, \\ u_{v}\left(t_{0}\right)=B_{\nu}\left(u\left(t_{0}\right), u\right), & v \in M\end{cases}
$$

Our main result extends Theorem 3.1 of [3] to infinite systems and it also improves [6] and Theorem 1.1 of [1]. The ideas contained in the proof of our main result are related to those of [7].

\section{Definitions and preliminaries}

We say that a partially ordered set (poset) $X$ is a lattice if $\sup \left\{x_{1}, x_{2}\right\}$ and $\inf \left\{x_{1}, x_{2}\right\}$ exist for all $x_{1}, x_{2} \in X$. A lattice $X$ is complete when each nonempty subset $Y \subset X$ has its supremum and infimum in $X$. In particular, every complete lattice has a maximum and a minimum.

In a poset $X$ we define for each $a, b \in X$, with $a \leq b$, the interval

$$
[a, b]:=\{x \in X: a \leq x \leq b\}
$$

The following result is the well-known Tarski fixed point theorem (see [12]).

THEOREM 2.1. Every nondecreasing mapping $G: X \rightarrow X$ on a complete lattice $X$ has a minimal and a maximal fuxed point, $x_{*}$ and $x^{*}$ respectively. Moreover,

$$
x_{*}=\min \{x \in X: G x \leq x\} \text { and } x^{*}=\max \{x \in X: x \leq G x\} \text {. }
$$

Let $M$ be an arbitrary index set. An element $x:=\left(x_{v}\right)_{\nu \in M}$ of $\mathbb{R}^{M}$ is denoted by $x:=\left(x_{\nu}, x^{\nu}\right)$ where $x^{\nu} \in \mathbb{R}^{M \backslash(\nu)}$. If $x, y \in \mathbb{R}^{M}$ we define the partial ordering

$$
x \leq y \quad \text { if and only if } \quad x_{v} \leq y_{\nu} \text { for all } v \in M
$$

We consider the Banach space

$$
l_{\infty}(M)=\left\{x:=\left(x_{v}\right)_{v \in M} \in \mathbb{R}^{M}:\|x\|:=\sup _{\nu \in M}\left|x_{v}\right|<+\infty\right\},
$$

and for the interval $I=\left[t_{0}, t_{1}\right]$ we define $C\left(I, l_{\infty}(M)\right)$ as the Banach space of all continuous functions $u: I \rightarrow l_{\infty}(M)$ with the norm $\|u\|_{0}=\sup \{\|u(t)\|: t \in I\}$, and we define the partial ordering, $u \leq v$ if and only if $u(t) \leq v(t)$ for all $t \in I$.

The following fixed point theorem is essentially Theorem 4 of [8] (see also Remark 6.3 in [8]). 
THEOREM 2.2. Let $a, b \in \mathbb{R}^{M}$, with $a \leq b$, and $f:=\left(f_{v}\right)_{v \in M}:[a, b] \rightarrow \mathbb{R}^{M}$ be a function such that $f(a) \leq a$ and $b \leq f(b)$. Suppose that $f$ satisfies the following properties for each $v \in M$ and for each $x \in[a, b]$ :

(i) The function $f_{v}\left(\cdot, x^{v}\right)$ is u.s.c. on the right and l.s.c. on the left on $\left[a_{v}, b_{v}\right]$, that is,

$$
\limsup _{y \rightarrow x_{v}^{-}} f_{v}\left(y, x^{\nu}\right) \leq f_{v}\left(x_{v}, x^{\nu}\right) \leq \liminf _{y \rightarrow x_{v}^{+}} f_{v}\left(y, x^{\nu}\right) .
$$

(ii) The function $f$ is quasimonotone, that is, $f_{v}\left(x_{\nu}, \cdot\right)$ is nondecreasing on $\left[a^{\nu}, b^{\nu}\right]$. Then the function $f$ has a minimal fixed point, $x_{*} \in[a, b]$, and a maximal fixed point, $x^{*} \in[a, b]$, and moreover they satisfy the properties

$$
\begin{aligned}
& x_{*}=\min \{x \in[a, b]: f(x) \leq x\}, \\
& x^{*}=\max \{x \in[a, b]: x \leq f(x)\} .
\end{aligned}
$$

REMARK 1. Theorem 2.2 extends to quasimonotone maps defined in arbitrary product spaces some earlier fixed point theorems by Hu and Schmidt, [9, 11], for quasimonotone maps defined in $\mathbb{R}^{n}$ and sequence spaces, respectively.

To end this section we introduce the classical concept of a lower solution of the scalar initial value problem

$$
u^{\prime}(t)=h(t, u(t)), \quad \text { for a.e. } t \in\left[t_{0}, t_{1}\right] ; \quad u\left(t_{0}\right)=A,
$$

with $h$ a Carathéodory function, and a function $\alpha \in A C\left(\left[t_{0}, t_{1}\right]\right)$, that is, the set of the absolutely continuous functions on the interval $\left[t_{0}, t_{1}\right]$, that satisfies the following inequalities:

$$
\alpha^{\prime}(t) \leq h(t, \alpha(t)), \quad \text { for a.e. } t \in\left[t_{0}, t_{1}\right] ; \quad \alpha\left(t_{0}\right) \leq A .
$$

The concept of an upper solution is given by reversing the previous inequalities. A solution of such a problem will be a function that is both a lower and an upper solution.

\section{Main result}

In this section we study the problem

$$
\begin{cases}u_{v}^{\prime}(t)=g_{v}(t, u(t), u) & \text { for a.a. } t \in I:=\left[t_{0}, t_{1}\right], v \in M, \\ u_{v}\left(t_{0}\right)=B_{v}\left(u\left(t_{0}\right), u\right), & v \in M,\end{cases}
$$

assuming that $g:=\left(g_{v}\right)_{v \in M}: I \times l_{\infty}(M) \times C\left(I, l_{\infty}(M)\right) \rightarrow l_{\infty}(M)$ and $B:=$ $\left(B_{v}\right)_{v \in M}: l_{\infty}(M) \times C\left(I, l_{\infty}(M)\right) \rightarrow l_{\infty}(M)$ satisfy for each $v \in M$ the following list of hypotheses which we will denote by $(\mathscr{A})$ : 
(g0) For all $u=\left(u_{v}, u^{v}\right) \in C\left(I, l_{\infty}(M)\right)$ and all $z \in \mathbb{R}$ the function $t \rightarrow$ $g_{v}\left(t, z, u^{v}(t), u\right)$ is Lebesgue measurable.

(g1) For a.a. $t \in I$ and for all $x=\left(x_{v}, x^{\nu}\right) \in l_{\infty}(M)$ and $u \in C\left(I, l_{\infty}(M)\right)$ the function $g_{v}\left(t, x_{v}, \cdot, u\right)$ is nondecreasing and

$$
\limsup _{y \rightarrow x_{v}^{-}} g_{v}\left(t, y, x^{\nu}, u\right) \leq g_{v}\left(t, x_{v}, x^{\nu}, u\right) \leq \liminf _{y \rightarrow x_{v}^{+}} g_{v}\left(t, y, x^{v}, u\right) .
$$

(g2) For a.a. $t \in I$ and for all $x \in l_{\infty}(M)$ the function $g_{v}(t, x, \cdot)$ is nondecreasing.

(g3) There exist $p, q, r \in L_{+}^{1}(I)$ such that for a.a. $t \in I$ and for all $x \in l_{\infty}(M)$ and $u \in C\left(I, l_{\infty}(M)\right)$ we have $\|g(t, x, u)\| \leq p(t)\|x\|+q(t)\|u\|_{0}+r(t)$.

(g4) $\|p\|_{L^{1}}+\|q\|_{L^{1}}<1$.

(B0) For each $x \in l_{\infty}(M)$ the operator $B_{v}(x, \cdot)$ is nondecreasing.

(B1) For all $x=\left(x_{\nu}, x^{\nu}\right) \in l_{\infty}(M)$ and $u \in C\left(I, l_{\infty}(M)\right)$ the function $B_{v}\left(x_{\nu}, \cdot, u\right)$ is nondecreasing and

$$
\limsup _{y \rightarrow x_{\nu}^{-}} B_{v}\left(y, x^{\nu}, u\right) \leq B_{v}\left(x_{v}, x^{\nu}, u\right) \leq \liminf _{y \rightarrow x_{\nu}^{+}} B_{v}\left(y, x^{\nu}, u\right) .
$$

(B2) There exist $a, b \in l_{\infty}(M)$, with $a \leq b$, such that for all $u \in C\left(I, l_{\infty}(M)\right)$

$$
a \leq B(a, u) \quad \text { and } \quad B(b, u) \leq b .
$$

DEFINITION 1. We say that $u=\left(u_{v}\right)_{v \in M} \in C\left(I, L_{\infty}(M)\right)$ is a solution of Problem (3.1) if it satisfies $u_{v} \in A C(I)$ for all $v \in M$ and

$$
\begin{cases}u_{v}^{\prime}(t)=g_{v}(t, u(t), u), & \text { for a.a. } t \in I, v \in M, \\ u_{\nu}\left(t_{0}\right)=B_{v}\left(u\left(t_{0}\right), u\right), & v \in M .\end{cases}
$$

Now we are ready to present our main result.

THEOREM 3.1. Assume the list of hypotheses $(\mathscr{A})$ is satisfied. Then Problem (3.1) has a minimal and a maximal solution in the set

$$
Y=\left\{u \in C\left(I, L_{\infty}(M)\right): a \leq u\left(t_{0}\right) \leq b\right\} .
$$

PROOF. We prove the existence of the maximal solution in $Y$ since the existence of the minimal solution is proved by dual arguments.

For each $u \in C\left(I, l_{\infty}(M)\right)$ we define the operator

$$
N(u):=\text { the maximal fixed point in }[a, b] \text { of function } B(\cdot, u) .
$$

Operator $N$ is well defined by Hypotheses (B1), (B2) and Theorem 2.2. Moreover, from (B0) and (2.2) it follows easily that $N$ is nondecreasing. 
Now, we define

$$
\begin{gathered}
R:=\frac{\max \{\|a\|,\|b\|\}+\|r\|_{L^{1}}}{1-\left(\|p\|_{L^{1}}+\|q\|_{L^{1}}\right)}, \quad h(t)=R[p(t)+q(t)]+r(t), \\
C=\left\{w: I \rightarrow \mathbb{R}: w \in[-R, R],|w(s)-w(t)| \leq\left|\int_{s}^{t} h(r) d r\right| \forall t, s \in I\right\}
\end{gathered}
$$

and $X=\prod_{\nu \in M} C$. Clearly $X \subset C\left(I, l_{\infty}(M)\right)$ and we consider for each $\nu \in M$ the operator $G_{v}: X \rightarrow C$ defined for each $v=\left(v_{v}, v^{\nu}\right) \in X$ as the maximal solution of the scalar initial value problem

$$
\left\{\begin{array}{l}
z^{\prime}(t)=g_{v}^{v}(t, z(t)), \quad \text { for a.a. } t \in I, \\
z\left(t_{0}\right)=N_{v}(v)
\end{array}\right.
$$

where the scalar function $g_{v}^{v}: I \times \mathbb{R} \rightarrow \mathbb{R}$ is defined for all $(t, z) \in I \times \mathbb{R}$ as

$$
g_{v}^{v}(t, z)=g_{v}\left(t, z, v^{v}(t), v\right)
$$

Claim 1. $G_{v}: X \rightarrow C$ is well defined.

For each $v \in M$ and $v=\left(v_{\nu}, v^{\nu}\right) \in X$ we consider the functions

$$
\beta(t)=(1+R) \mathrm{e}^{\int_{t_{0}}^{t}([p(s)+q(s)] R+r(s)) d s}-1
$$

and $\alpha(t)=-\beta(t)$ for all $t \in I$. It is easy to verify that $\beta(t) \geq R \geq-R \geq \alpha(t)$ for all $t \in I$ and that $\alpha$ and $\beta$ are lower and upper solutions, respectively, for (3.3). Moreover, by Hypotheses (g0), (g1) and (g3) the function $g_{v}^{v}$ satisfies Conditions 1-3 of [10, Theorem 2.4] and thus there exists the maximal solution, $z^{*}$, of (3.3) in $[\alpha, \beta]$, which moreover satisfies

$$
z^{*}=\max \left\{z \in[\alpha, \beta]: z^{\prime}(t) \leq g_{v}^{v}(t, z(t)) \text { a.e. } I, z\left(t_{0}\right) \leq N_{v}(v)\right\} .
$$

Furthermore it is easy to check that any solution $z$ of Problem (3.3) satisfies that $\|z\| \leq R$ and therefore $z \in[\alpha, \beta]$. Thus $z^{*}$ is the maximal solution of (3.3) (not only in $[\alpha, \beta])$.

CLAIM 2. $X$ is a complete lattice.

Since $X=\prod_{\nu \in M} C$ it is enough to prove that $C$ is a complete lattice. Given a nonempty subset $Y \subset C$ it is easy to prove that $w_{*}(t):=\inf \{w(t): w \in Y\}$ and $w^{*}(t):=\sup \{w(t): w \in Y\}$ for all $t \in I$, are the infimum and the supremum of $Y$ in $C$, respectively.

ClaIM 3. $G:=\left(G_{\nu}\right)_{\nu \in M}: X \rightarrow X$ is nondecreasing. 
By using Hypotheses (g1), (g2), the fact that $N$ is nondecreasing and property (3.4), it is easy to prove that $G_{\nu}: X \rightarrow C$ is nondecreasing for all $v \in M$.

By Claims 2 and 3, the Tarski fixed point theorem ensures that $G$ has the maximal fixed point, $u^{*} \in X$, which satisfies

$$
u^{*}=\max \{u \in X: u \leq G u\} .
$$

ClaIM 4. The maximal fixed point of $G, u^{*}$, is the maximal solution in $Y$ of (3.1).

Clearly, $u^{*}$ is a solution in $Y$ of (3.1). Let $u$ be another solution in $Y$ of (3.1). Then it is easy to verify that $u \in X$ and $u \leq G u$. Therefore from (3.5) it follows that $u \leq u^{*}$ and thus $u^{*}$ is the maximal solution of Problem (3.1).

COROLlaRY 3.2. Assume Hypotheses (g0)-(g4), (B0), (B1) and

$$
\text { ( } \overline{\mathrm{B} 2}) \limsup _{\|x\| \rightarrow \infty} \frac{\|B(x, u)\|}{\|x\|}<1 \text {, uniformly at } u \in C\left(I, l_{\infty}(M)\right) \text {. }
$$

Then (3.1) has a minimal and a maximal solution.

ProOF. Let

$$
\limsup _{\|x\| \rightarrow \infty} \frac{\|B(x, u)\|}{\|x\|}=c<1 .
$$

By choosing in the definition of lim sup the value of $\epsilon=(1-c) / 2>0$, we have that there exists $K>0$ such that for all $u \in C\left(I, l_{\infty}(M)\right)$ and all $d>0$, it holds that

$$
\|B(x, u)\|<\frac{c+1}{2}\|x\|+d \text { for all } x \in \mathbb{R}^{M} \text { such that }\|x\|>K .
$$

Therefore, by taking $d>K(1-c) / 2$, we arrive at the fact that $a=\left(a_{v}\right)_{v \in M}$ and $b=\left(b_{v}\right)_{v \in M}$ defined as

$$
a_{\nu}=-\frac{2 d}{1-c} \quad \text { and } \quad b_{v}=\frac{2 d}{1-c}
$$

satisfy the properties imposed in Condition (B2).

Thus Theorem 3.1 ensures the existence of the extremal solutions, $x_{*}$ and $x^{*}$, in the set $Y=\left\{u \in C\left(I, L_{\infty}(M)\right): a \leq u\left(t_{0}\right) \leq b\right\}$.

Moreover, if $u$ is any solution of Problem (3.1), in particular, $u\left(t_{0}\right)=B\left(u\left(t_{0}\right), u\right)$. If $\left\|u\left(t_{0}\right)\right\|>K$ then, from the previous arguments, we have that

$$
\left\|u\left(t_{0}\right)\right\|=\left\|B\left(u\left(t_{0}\right), u\right)\right\| \leq \frac{c+1}{2}\left\|u\left(t_{0}\right)\right\|+d
$$

and then $\left\|u\left(t_{0}\right)\right\| \leq 2 d /(1-c)$. Therefore, $a \leq u\left(t_{0}\right) \leq b$ and we have that $u \in Y$ and thus $x_{*} \leq u \leq x^{*}$. Then $x_{*}$ and $x^{*}$ are the extremal solutions. 
REMARK 2. If we define lower and upper solutions, $\alpha$ and $\beta$, for (3.1) as in [5, page 47] in the case of one equation, and we assume Hypotheses (g0)-(g2), (B0), (B1) and

(B) There exists $h \in L_{+}^{1}(I)$ such that for all $u \in[\alpha, \beta]$

$$
\|g(t, x, u)\| \leq h(t) \quad \text { for a.a. } t \in I \text { and all } \alpha(t) \leq x \leq \beta(t),
$$

we deduce from [7, Theorem 4.1] the existence of extremal solutions in the order interval determined by the lower and the upper solutions.

REMARK 3. The example given in [1, Section 5], which is a modification of the well-known example of Dieudonné, shows that Theorem 3.1 is not true, in the case $M=\mathbb{N}$, when we replace $l_{\infty}(\mathbb{N})$ by $c_{0}(\mathbb{N})$, the set of the sequences that converge to zero.

\section{References}

[1] D. C. Biles and E. Schechter, "Solvability of a finite or infinite system of discontinuous quasimonotone differential equations,", Proc. Amer. Math. Soc. 128 (2000) 3349-3360.

[2] A. Cabada, M. R. Grossinho and F. Minhós, "On the solvability of some discontinuous third order nonlinear differential equations with two point boundary conditions", J. Math. Anal. Appl. 285 (2003) 174-190.

[3] A. Cabada and S. Heikkilä, "Existence results for discontinuous functional differential systems", submitted.

[4] A. Cabada and S. Heikkilä, "Existence of solutions of third-order functional problems with nonlinear boundary conditions", ANZIAM J. 46 (2004) 33-44.

[5] S. Carl and S. Heikkilä, Nonlinear differential equations in ordered spaces (Chapman and Hall/CRC, Boca Raton, FI, USA, 2000).

[6] A. Chaljub-Simon and P. Volkmann, "Un théorème d'existence et de comparaison pour des équations différentielles dans les espaces de fonctions bornées", C. R. Acad. Sci. Paris Sér. I Math. 311 (1990) 515-517.

[7] J. A. Cid, "On extending existence theory from scalar ordinary differential equations to infinite quasimonotone systems of functional equations", Proc. Amer. Math. Soc. 133 (2005) 2661-2670.

[8] J. Guillerme, "Intermediate value theorems and fixed point theorems for semi-continuous functions in product spaces", Proc. Amer. Math. Soc. 123 (1995) 2119-2122.

[9] S. Hu, "Fixed points for discontinuous quasi-monotone maps in $\mathbb{R}^{n}$ ", Proc. Amer. Math. Soc. 104 (1988) 1111-1114.

[10] E. Liz and R. L. Pouso, "Existence theory for first order discontinuous functional differential equations", Proc. Amer. Math. Soc. 130 (2002) 3301-3311.

[11] S. Schmidt, "Fixed points for discontinuous quasi-monotone maps in sequence spaces", Proc. Amer. Math. Soc. 115 (1992) 361-363.

[12] A. Tarski, "A lattice-theoretical fixed point theorem and its applications", Pacific J. Math. 5 (1955) 285-309. 